\title{
Archaeometry, an interdisciplinary approach
}

\author{
Peter Vandenabeele
}

Published online: 4 January 2007

(C) Springer-Verlag 2007

Since the 1950s, interest in the preservation and study of our cultural heritage has grown substantially. Apart from the aesthetic value derived from artworks, people are interested in the historical background behind such objects: they want to know how the objects were used, their historical context, and the changes or degradations they have undergone since they were used. Archaeometry is an interdisciplinary research field that covers aspects of analytical chemistry and spectroscopy, (bio)organic chemistry, electrochemistry, physics, conservation science, museology, anthropology, history, history of art and archaeology, amongst other fields. Often, research in this field attempts to investigate the degradation, provenance or relationships between several artefacts. Work in this area of research is quite challenging, since vulnerable and precious artefacts are involved and the analysts must use all of their skills to gather the information while performing direct investigations or working on microsamples. Research in archaeometry often requires the development, optimisation or adaptation of sensitive analytical techniques which can then be used to investigate antique objects or precious artefacts. Nowadays, a whole set of analytical approaches are available for investigations of artworks. This choice may be confusing to people who are new to this field, since an understanding of the advantages and possible drawbacks of all these techniques, or when to apply each technique, is required.

Unfortunately, some people seem to consider this area of research as merely as an innocent hobby pursued by some scientists because it involves working with beautiful artworks. However, good skills in analytical chemistry are

P. Vandenabeele $(\bowtie)$

Department of Analytical Chemistry, Ghent University,

Proeftuinstraat 86 ,

9000 Ghent, Belgium

e-mail: peter.vandenabeele@UGent.be vital when working with unique artefacts that cannot be damaged during investigations. It is necessary to maximise the information gathered, despite the restrictions placed on the investigation by interferences, limited sample material, the inability to repeat measurements, and the often short period of time that is given to the researcher in order to study the artefact. If possible, sample destruction should be avoided, so that several analytical techniques can be applied to the same sample. Many hours of hard work and patience are often involved in such research. However, it is definitely amazing to work with these beautiful artworks.

Talking to scientists who work in archaeometry, it is remarkable how enthusiastic they are. One colleague tried to convince art historians of the beauty of a spectrum with well-defined peaks and a good signal-to-noise ratio. A conservator also shared some philosophical ideas with me about the occurrence of cosmic rays-spikes - in my detector. Aside from the added scientific value of interdisciplinary science, research performed in archaeometry yields an additional social bonus! Dear reader, in this special issue of Analytical and Bioanalytical Chemistry, we would like to share our enthusiasm with you.

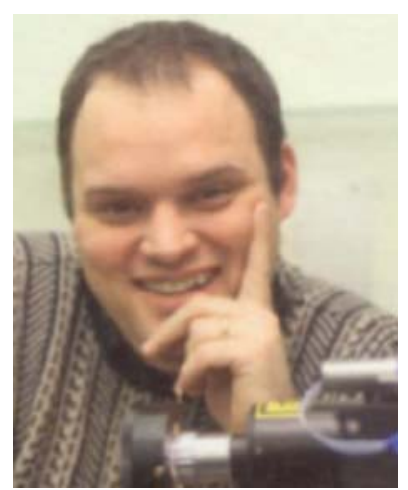

Peter Vandenabeele

works as a post-doctoral fellow at Ghent University, where he performs research in analytical Raman spectroscopy 